\title{
Treatment of lymphangioleiomyomatosis: building evidence in orphan diseases
}

\author{
Vincent Cottin ${ }^{1,2}$
}

Affiliations: ${ }^{1}$ Hospices Civils de Lyon, Hôpital Louis Pradel, Service de pneumologie - Centre de référence national des maladies pulmonaires rares, Lyon, and ${ }^{2}$ Université de Lyon, Université Claude Bernard Lyon 1 , INRA, UMR754 INRA-Vetagrosup EPHE IFR 128, Lyon, France.

Correspondence: V. Cottin, Hôpital Louis Pradel, Claude Bernard Lyon 1 University, 28 Avenue du Doyen Lepine, 69677 Lyon, France. E-mail: vincent.cottinachu-lyon.fr

0

@ERSpublications

In orphan disease, a negative trial contributes to building evidence and excluding ineffective therapy from practice http://ow.ly/tvANk

Lymphangioleiomyomatosis (LAM) is a rare (orphan) lung disease, in which the lung parenchyma is progressively replaced by cysts associated with a proliferation of immature smooth muscle cells and perivascular epithelioid cells (so-called LAM cells) around lymphatic vessels [1, 2]. LAM can be sporadic, almost exclusively affecting females, or can be associated with tuberous sclerosis complex (TSC) where it affects $30-50 \%$ of adult females, and less frequently males [3-6].

Patients with LAM most commonly present with relapsing pneumothorax and dyspnoea on exertion, and may have angiomyolipomas (benign tumours) of the kidneys and, occasionally, chylothorax or chylous ascites. Computed tomography of the chest shows characteristic multiple, round thin-walled cysts evenly distributed throughout the lung parenchyma [7], possibly associated with pneumothorax, pleural effusion, lymphadenopathies or lymphangiomas. Over time the disease may progress to chronic obstructive respiratory failure that may require lung transplantation. Pulmonary hypertension is very rare [8]. Diagnostic criteria and management guidelines have been proposed by a task force of the European Respiratory Society. Furthermore, the serum level of vascular endothelial growth factor (VEGF)-D, a major growth factor for lymphatic vessels, may further contribute to the noninvasive diagnosis of LAM if elevated [9].

For many years, treatment of LAM has been mostly supportive, including bronchodilators, supplemental oxygen (if needed), management of complications (especially pneumothorax) and lung transplantation. With a high variability of disease progression [10], many patients with LAM do not need a treatment intervention, whereas a minority of patients do progress to respiratory failure. In 2000, a major breakthrough in the understanding of the pathophysiology of the disease led to a novel treatment approach 10 years later [11]. Based on the observation that pulmonary LAM is present in patients with TSC [4], the group of E.P. Henske identified that LAM cells carry bi-allelic mutations within the TSC2 gene, a tumour suppressor gene that encodes tuberin $[12,13]$, with similar mutations being found in LAM cells from the lung tissue and abnormal smooth muscle cells in angiomyolipomas [12]. TSC2 mutations are responsible for the constitutive activation of the phosphoinositol-3-kinase/Akt/mammalian target of rapamycin (mTOR)/S6-kinase pathway (mTORC1), which in turn enhances the proliferation of LAM cells [14].

This has led to several clinical trials demonstrating a beneficial effect of mTOR inhibitors (sirolimus and everolimus) in benign tumours associated with TSC, such as angiomyolipomas (also observed in sporadic LAM) $[15,16]$ and subependymal giant cell astrocytomas [17-19]. Sirolimus therapy has been demonstrated

Received: Feb 052014 | Accepted: Feb 062014

Support statement: This work has been supported, in part, by a grant in 2009 from Comité National contre les Maladies Respiratoires (CNMR) - Fondation du Souffle.

Conflict of interest: Disclosures can be found alongside the online version of this article at www.erj.ersjournals.com

Copyright @ERS 2014 
in a placebo-controlled randomised trial to stabilise lung function compared with placebo in 89 patients with pulmonary LAM (sporadic or associated with TSC) [20]. The safety and efficacy of everolimus, another mTOR inhibitor with a different pharmacokinetic profile, has been evaluated in an open-label phase II study (www.clinicaltrials.gov, identifier NCT01059318) that was recently completed in patients with LAM. mTOR inhibitors also appear effective on chylous effusion [21] and lymphangioleiomyomas [22, 23] in small observational studies. As they are not currently approved for LAM, their compassionate use should be restricted to centres expert in LAM. Of note, mTOR inhibitors, which were developed in renal transplantation, are now used in a wide range of conditions associated with cell proliferation including a variety of cancers (renal cell carcinoma, neuroendocrine tumours of pancreatic origin and breast cancer), and it is likely that the experience of clinicians and knowledge about the long-term safety profile will increase greatly in coming years.

However, mTOR inhibitors are not the panacea to treat pulmonary LAM. The benefit of therapy is only suspensive, and progression of disease resumes following discontinuation of the drug [15, 20]. In vitro, mTOR inhibitors only partially reverse abnormalities observed in LAM cells, at least in part, due to mTORC1 feedback loops [24]. mTOR inhibitors are responsible for many adverse events (including mouth ulcers, diarrhoea, nausea, increased blood cholesterol levels, skin rash and swelling of the extremities), most of which are manageable, yet preclude the long-term use of the drugs in a proportion of patients. A current approach is to use lower doses of mTOR inhibitors to improve their safety profile [25]; however, the benefit/risk ratio of lower doses has not been evaluated. Therefore, further investigation is needed to identify alternative pathways and pathogenic mechanisms involved in LAM and to develop novel therapies.

The origin of LAM cells is unknown [24], but the current hypothesis is that they might originate from the uterus, where HMB45-positive epithelioid-shaped LAM cells can be found in $90 \%$ of the patients by histological and immunohistological analyses [26]. It is now well established that cells invade the lymphatic vessels and cell clusters spread to selected distant sites, especially the lung and kidney [24]. LAM cells (harbouring loss of heterozygosity of the TSC2 locus) can be found in a variety of body fluids including the blood and lymphatic system [27-29]. LAM cells further induce angiogenesis and lymphangiogenesis, and exhibit metabolic switch toward aerobic glycolysis [30]. Therefore, LAM is currently considered as a lowgrade metastatic neoplasm [31], a concept that may foster innovation in LAM research [30]. This further supports the development of alternative approaches to the current use of mTOR inhibitors that mostly target cell proliferation, with a lesser or no effect on cell adhesion, migration, motility, survival, resistance to anoikis, and release of pathogenic mediators including proteases.

Matrix metalloproteinases (MMPs), which can degrade extracellular matrix, are produced by LAM cells and have been implicated in the formation of lung cysts in LAM, especially MMP-1, MMP-2, MMP-9 and MMP-14, which are elevated in the serum and/or expressed in lung tissue adjacent to cysts [32-35]. Furthermore, MMPs are known to affect cell growth, invasion, inflammation and angiogenesis [36]. These observations provided a strong rationale for inhibiting MMP activity in LAM patients.

In the current issue of the European Respiratory Journal, CHANG et al. [37] report the results of a randomised, monocentric, double-blind trial comparing the MMP inhibitor doxycycline with placebo on the progression of LAM over 2 years in patients with moderately severe disease. Doxycycline, a tetracycline antibiotic, is the only licensed drug that exhibits inhibition of MMP-1, MMP-2 and MMP-9 [38] which are implicated in LAM and, thus, prompted the choice of doxycycline for this trial. In addition, some suggestion for a benefit of doxycycline in LAM had come from a previous case report and observational studies [39-41]. Difficulties were experienced for patient inclusion and retention in the trial [37], therefore decreasing the power of the study. However, 23 patients were randomised; 17 completed 1 year of treatment and 15 completed 2 years of treatment. No significant difference was observed between the doxycycline and placebo arms in the mean decline in forced expiratory volume in $1 \mathrm{~s}$, the primary end-point of the study. No effect was observed on any of the secondary end-points (vital capacity, gas transfer, shuttle walk distance and quality of life), although inhibition of MMP production by doxycycline therapy was confirmed by lower levels of active MMP-9 measurements in urine (serum levels of MMP-2 and MMP-9 were unchanged). Doxycycline was generally well tolerated.

Although the trial is negative, the authors should be commended for conducting such a challenging study in a very rare condition, as it is only the second randomised trial conducted to date in patients with LAM (the first being on sirolimus [20]), and for exploring pathways other than mTOR in LAM treatment. Even if negative, a study that addresses a relevant hypothesis, such as the present one, is useful for patients and their physicians. Of course one may question whether similar results would have been obtained had the study reached complete enrolment with better balance in the severity of lung function impairment [40], or had been conducted in a population of LAM patients with greater severity, more rapid decline in lung function, and/or with a higher proportion of pre-menopausal patients (who admittedly have more progressive disease). Given the absence of any signal on end-points, this study does provide sufficient evidence that 
doxycycline, a drug that has been taken off-label by many LAM patients, should no longer be used in LAM. The results by CHANG et al. [37] also remind us that observations in case reports or observational studies may not be confirmed in randomised trials. Excluding therapies from our practice that are now demonstrated to be ineffective (such as progesterone derivatives or more invasive hormonal interventions in LAM [1, 42, 43], or immunosuppressive therapy in idiopathic pulmonary fibrosis [44]), significantly contributes to improving patient management.

This study illustrates the typical challenges of conducting studies in rare diseases [45-47]. Inclusion was limited by the rarity of the disease, the need for patients to travel to the expert centre, and by a large proportion of the patients already receiving doxycycline or mTOR inhibitors. Some patients dropped out the study later to opt for an mTOR inhibitor, or could not be analysed due to pneumothorax [37]. Some of these difficulties were shared by previous studies conducted in this and other rare diseases. LAM research is currently very active, with several clinical trials ongoing or in preparation using aromatase inhibitors or the agent fulvestrant (which targets the oestrogen receptor- $\alpha[30,48,49]$ ), targeting autophagy [50] or using simvastatin in combination with sirolimus [51, 52]. VEGF-D [53], or its receptor VEGFR-3, Rheb [30], integrins, etc., may also represent effective targets for therapy. In fact, there are now more potential treatment targets than can be practically tested given the very low prevalence of the disease and available resources.

As with previous trials in LAM [45], the study would not have been possible without the active involvement of patient associations, and the interaction of investigators with patients (some of whom are becoming expert patients) and patient associations [46]. What might particularly reflect patients' preferences in research is the presence of quality of life as an end-point in LAM studies [20,37]. Patient-reported outcome is increasingly being measured in clinical trials and registries [54], reflecting the aim of investigators and regulators to consider patients' symptoms and well-being as part of the management goal.

\section{References}

1 Johnson SR, Cordier JF, Lazor R, et al. European Respiratory Society guidelines for the diagnosis and management of lymphangioleiomyomatosis. Eur Respir J 2010; 35: 14-26.

2 Glasgow CG, El-Chemaly S, Moss J. Lymphatics in lymphangioleiomyomatosis and idiopathic pulmonary fibrosis. Eur Respir Rev 2012; 21: 196-206.

3 Moss J, Avila NA, Barnes PM, et al. Prevalence and clinical characteristics of lymphangioleiomyomatosis (LAM) in patients with tuberous sclerosis complex. Am J Respir Crit Care Med 2001; 164: 669-671.

4 Cudzilo CJ, Szczesniak RD, Brody AS, et al. Lymphangioleiomyomatosis screening in women with tuberous sclerosis. Chest 2013; 144: 578-585.

5 Muzykewicz DA, Black ME, Muse V, et al. Multifocal micronodular pneumocyte hyperplasia: computed tomographic appearance and follow-up in tuberous sclerosis complex. J Comput Assist Tomogr 2012; 36: 518-522. Ryu JH, Sykes AM, Lee AS, et al. Cystic lung disease is not uncommon in men with tuberous sclerosis complex. Respir Med 2012; 106: 1586-1590.

7 Avila NA, Dwyer AJ, Rabel A, et al. Sporadic lymphangioleiomyomatosis and tuberous sclerosis complex with lymphangioleiomyomatosis: comparison of ct features. Radiology 2007; 242: 277-285.

8 Cottin V, Harari S, Humbert M, et al. Pulmonary hypertension in lymphangioleiomyomatosis: characteristics in 20 patients. Eur Respir J 2012; 40: 630-640.

9 Young LR, Vandyke R, Gulleman PM, et al. Serum vascular endothelial growth factor-D prospectively distinguishes lymphangioleiomyomatosis from other diseases. Chest 2010; 138: 674-681.

10 Lazor R, Lauque D, Delaval P, et al. Predictors of rapid decline of FEV1 in 50 cases of pulmonary lymphangioleiomyomatosis followed for $\geqslant 1$ year. Am J Respir Crit Care Med 2000; 161: A15.

Cottin V, Archer F, Leroux C, et al. Milestones in lymphangioleiomyomatosis research. Eur Respir Rev 2011; 20: 3-6. Carsillo T, Astrinidis A, Henske EP. Mutations in the tuberous sclerosis complex gene TSC2 are a cause of sporadic pulmonary lymphangioleiomyomatosis. Proc Natl Acad Sci USA 2000; 97: 6085-6090.

$13 \mathrm{Yu} \mathrm{J}$, Astrinidis A, Henske EP. Chromosome 16 loss of heterozygosity in tuberous sclerosis and sporadic lymphangiomyomatosis. Am J Respir Crit Care Med 2001; 164: 1537-1540.

14 Yu J, Henske EP. mTOR activation, lymphangiogenesis, and estrogen-mediated cell survival: the "perfect storm" of pro-metastatic factors in LAM pathogenesis. Lymphat Res Biol 2010; 8: 43-49.

15 Bissler JJ, McCormack FX, Young LR, et al. Sirolimus for angiomyolipoma in tuberous sclerosis complex or lymphangioleiomyomatosis. N Engl J Med 2008; 358: 140-151.

16 Bissler JJ, Kingswood JC, Radzikowska E, et al. Everolimus for angiomyolipoma associated with tuberous sclerosis complex or sporadic lymphangioleiomyomatosis (EXIST-2): a multicentre, randomised, double-blind, placebocontrolled trial. Lancet 2013; 381: 817-824.

17 Franz DN, Leonard J, Tudor C, et al. Rapamycin causes regression of astrocytomas in tuberous sclerosis complex. Ann Neurol 2006; 59: 490-498.

18 Franz DN, Belousova E, Sparagana S, et al. Efficacy and safety of everolimus for subependymal giant cell astrocytomas associated with tuberous sclerosis complex (exist-1): a multicentre, randomised, placebo-controlled phase 3 trial. Lancet 2013; 381: 125-132.

19 Krueger DA, Care MM, Holland K, et al. Everolimus for subependymal giant-cell astrocytomas in tuberous sclerosis. N Engl J Med 2010; 363: 1801-1811.

20 McCormack FX, Inoue Y, Moss J, et al. Efficacy and safety of sirolimus in lymphangioleiomyomatosis. N Engl J Med 2011; 364: 1595-1606.

21 Moua T, Olson EJ, Jean HC, et al. Resolution of chylous pulmonary congestion and respiratory failure in lymphangioleiomyomatosis with sirolimus therapy. Am J Respir Crit Care Med 2012; 186: 389-390. 
22 Mohammadieh AM, Bowler SD, Silverstone EJ, et al. Everolimus treatment of abdominal lymphangioleiomyoma in five women with sporadic lymphangioleiomyomatosis. Med J Aust 2013; 199: 121-123.

23 Taveira-DaSilva AM, Hathaway O, Stylianou M, et al. Changes in lung function and chylous effusions in patients with lymphangioleiomyomatosis treated with sirolimus. Ann Intern Med 2011; 154: 797-292.

24 Henske EP, McCormack FX. Lymphangioleiomyomatosis - a wolf in sheep's clothing. J Clin Invest 2012; 122: 3807-3816.

25 Ando K, Kurihara M, Kataoka H, et al. The efficacy and safety of low-dose sirolimus for treatment of lymphangioleiomyomatosis. Respir Investig 2013; 51: 175-183.

26 Hayashi T, Kumasaka T, Mitani K, et al. Prevalence of uterine and adnexal involvement in pulmonary lymphangioleiomyomatosis: a clinicopathologic study of 10 patients. Am J Surg Pathol 2011; 35: $1776-1785$.

27 Crooks DM, Pacheco-Rodriguez G, DeCastro RM, et al. Molecular and genetic analysis of disseminated neoplastic cells in lymphangioleiomyomatosis. Proc Natl Acad Sci USA 2004; 101: 17462-17467.

28 Kumasaka T, Seyama K, Mitani K, et al. Lymphangiogenesis-mediated shedding of LAM cell clusters as a mechanism for dissemination in lymphangioleiomyomatosis. Am J Surg Pathol 2005; 29: 1356-1366.

29 Mitani K, Kumasaka T, Takemura H, et al. Cytologic, immunocytochemical and ultrastructural characterization of lymphangioleiomyomatosis cell clusters in chylous effusions of patients with lymphangioleiomyomatosis. Acta Cytol 2009; 53: 402-409.

30 El-Chemaly S, Henske EP. Towards personalised therapy of lymphangioleiomyomatosis: lessons from cancer. Eur Respir Rev 2014; 23: 30-35.

31 McCormack FX, Travis WD, Colby TV, et al. Lymphangioleiomyomatosis: calling it what it is: a low-grade, destructive, metastasizing neoplasm. Am J Respir Crit Care Med 2012; 186: 1210-1212.

32 Odajima N, Betsuyaku T, Nasuhara Y, et al. Matrix metalloproteinases in blood from patients with LAM. Respir Med 2009; 103: 124-129.

33 Chang WY, Cane JL, Blakey JD, et al. Clinical utility of diagnostic guidelines and putative biomarkers in lymphangioleiomyomatosis. Respir Res 2012; 13: 34.

34 Hayashi T, Fleming MV, Stetler-Stevenson WG, et al. Immunohistochemical study of matrix metalloproteinases (MMPs) and their tissue inhibitors (TIMPs) in pulmonary lymphangioleiomyomatosis (LAM). Hum Pathol 1997; 28: 1071-1078.

35 Matsui K, Takeda K, Yu ZX, et al. Role for activation of matrix metalloproteinases in the pathogenesis of pulmonary lymphangioleiomyomatosis. Arch Pathol Lab Med 2000; 124: 267-275.

36 Khokha R, Murthy A, Weiss A. Metalloproteinases and their natural inhibitors in inflammation and immunity. Nat Rev Immunol 2013; 13: 649-665.

37 Chang WYC, Cane JL, Kumaran M, et al. A 2-year randomised placebo-controlled trial of doxycycline for lymphangioleiomyomatosis. Eur Respir J 2014; 43: 1114-1123.

38 Castro MM, Kandasamy AD, Youssef N, et al. Matrix metalloproteinase inhibitor properties of tetracyclines: therapeutic potential in cardiovascular diseases. Pharmacol Res 2011; 64: 551-560.

39 Pimenta SP, Baldi BG, Kairalla RA, et al. Doxycycline use in patients with lymphangioleiomyomatosis: biomarkers and pulmonary function response. J Bras Pneumol 2013; 39: 5-15.

40 Baldi BG, Carvalho CRR. Doxycycline in lymphangioleiomyomatosis: not all questions are answered. Eur Respir J 2014 [In press DOI: 10.1183/09031936.00008114].

41 Moses MA, Harper J, Folkman J. Doxycycline treatment for lymphangioleiomyomatosis with urinary monitoring for mmps. N Engl J Med 2006; 354: 2621-2622.

42 Taveira-DaSilva AM, Hathaway OM, Moss J. Effect of progesterone (PG) and bisphosphonates (B) on bone mineral density (BMD) in patients with lymphangioleiomyomatosis (LAM). Am J Respir Crit Care Med 2004; $169:$ A225.

43 Johnson SR, Whale CI, Hubbard RB, et al. Survival and disease progression in UK patients with lymphangioleiomyomatosis. Thorax 2004; 59: 800-803.

44 Raghu G, Anstrom KJ, King TE Jr, et al. Prednisone, azathioprine, and N-acetylcysteine for pulmonary fibrosis. N Engl J Med 2012; 366: 1968-1977.

45 Ingelfinger JR, Drazen JM. Patient organizations and research on rare diseases. N Engl J Med 2011; 364: 1670-1671.

46 Spagnolo P, du Bois RM, Cottin V. Rare lung disease and orphan drug development. Lancet Respir Med 2013; 1: 479-487.

47 Kinder B, McCormack FX. Clinical trials for rare lung diseases: lessons from lymphangioleiomyomatosis. Lymphat Res Biol 2010; 8: 71-79.

48 Li C, Zhou X, Sun Y, et al. Faslodex inhibits estradiol-induced extracellular matrix dynamics and lung metastasis in a model of lymphangioleiomyomatosis. Am J Respir Cell Mol Biol 2013; 49: 135-142.

49 Shim B, Pacheco-Rodriguez G, Kato J, et al. Sex-specific lung diseases: effect of oestrogen on cultured cells and in animal models. Eur Respir Rev 2013; 22: 302-311.

50 Yu J, Parkhitko A, Henske EP. Autophagy: an "Achilles" heel of tumorigenesis in TSC and LAM. Autophagy 2011; 7: 1400-1401.

51 Goncharova EA, Goncharov DA, Li H, et al. mTORC2 is required for proliferation and survival of TSC2-null cells. Mol Cell Biol 2011; 31: 2484-2498.

52 Atochina-Vasserman EN, Goncharov DA, Volgina AV, et al. Statins in lymphangioleiomyomatosis. Simvastatin and atorvastatin induce differential effects on tuberous sclerosis complex 2-null cell growth and signaling. Am J Respir Cell Mol Biol 2013; 49: 704-709.

53 Seyama K, Kumasaka T, Souma S, et al. Vascular endothelial growth factor-D is increased in serum of patients with lymphangioleiomyomatosis. Lymphat Res Biol 2006; 4: 143-152.

54 Nurok M, Eslick I, Carvalho CR, et al. The international LAM registry: a component of an innovative web-based clinician, researcher, and patient-driven rare disease research platform. Lymphat Res Biol 2010; 8: 81-87. 